\title{
Faster and More Cost-effective Way to Success in MEMS R\&D by Open Collaboration in Tohoku University
}

\author{
Shuji Tanaka* \\ Department of Robotics and Microsystem Integration Center, Tohoku University \\ 6-6-01 Aramaki Aza Aoba, Aoba-ku, Sendai, Miyagi 908-8579, Japan
}

(Received January 22, 2018; accepted February 27, 2018)

Keywords: micro-electromechanical systems (MEMS), collaboration, tactile sensor

In this paper, "Open Collaboration" is defined as research and development (R\&D) in a value/supply chain composed of different organizations including companies, universities and research institutes. If Open Collaboration is defined like this, it is not new, but it was not working well in Japanese universities. This paper first explains the scheme of Open Collaboration of micro-electromechanical systems (MEMS) in Tohoku University. Then, the project of integrated tactile sensors is described as a successful example of Open Collaboration. The purpose of this paper is to promote Open Collaboration in the field of MEMS by introducing our practices.

\section{Introduction}

The phrase "Open Collaboration", which is the topic of this special issue organized by Prof. Masayoshi Esashi, is less common than the similar phrase "Open Innovation", which has often been used in Japan recently. The former phrase mainly represents what is going at the sites of research and development $(R \& D)$, while the latter phrase focuses more on the outcome from such R\&D activity, but the two phrases share a similar concept.

Conventionally, a major part of the value chain of $R \& D$ is closed in a company or a cooperative group in Japan. Universities and research institutes were outside the value/supply chain of R\&D. The similar situation in the manufacturing sector referred to as "Vertical Integration" has changed in the past 20 years, especially in the semiconductor and electronics industry. "Horizontal Specialization" may be a good phrase to express this trend in comparison with Vertical Integration. As an example of Horizontal Specialization, fabless companies and foundries are successful in the field of micro-electromechanical systems (MEMS), which is my specialty.

By Horizontal Specialization, the speed of manufacturing business has increased. Accordingly, the speed of R\&D and innovation must have also been increased, which is a motivation and merit of Open Collaboration. The value/supply chain of R\&D is now opened outside and composed of technologies, knowledges, ideas and/or facilities in different

*Corresponding author: e-mail: tanaka@mems.mech.tohoku.ac.jp

http://dx.doi.org/10.18494/SAM.2018.1914 
organizations including universities and research institutes. It is often more rapid and costeffective to outsource a part of $R \& D$ to a partner which is better at the technology to be developed than to develop everything internally.

This style of $R \& D$ has been successful for a long time in European research institutes such as the Fraunhofer Institutes (Germany) and IMEC (Belgium). On the other hand, Open Collaboration is less successful and still on the way to becoming popular in Japan. One of the reasons may be that Japanese companies are sticking to the past successful experiences with Vertical Integration. Another reason may be that the values of information and technology, which will be provided by R\&D partners, are underestimated, which is reflected by the budget for collaboration. However, global competitiveness in $R \& D$ as well as manufacturing business is becoming more intense, and Open Collaboration is now an important keyword.

\section{Open Collaboration of MEMS in Tohoku University}

\subsection{Scheme of Open Collaboration}

Microsystem Integration Center together with S. Tanaka Laboratory is now promoting Open Collaboration of MEMS. Microsystem Integration Center is composed of R\&D division and "Hands-on Access Facility" division. The former division is mainly responsible for R\&D with customers, i.e., companies with collaborative research contracts with us, as well as governmentfunded R\&D projects. The latter division is mainly responsible for the open access service and maintenance of our facility, and strongly supported by the "Nanotechnology Platform Project" under the Ministry of Education, Culture, Sports, Science and Technology (MEXT), Japan. The mission of each division is clearly defined, but two divisions are working synergistically to obtain high satisfaction of customers and users. This is one of our strengths.

Most of our collaborative R\&D projects are carried out with a conventional scheme, i.e., exclusive collaboration between a customer and us. However, we also have "more opened" collaboration projects. Note that the word "open" does not mean that the output, information or intellectual properties are opened to the outside of the project. I hope that the meaning of open in this context is clear from the Introduction. What is opened outside is the value/supply chain of R\&D. In the following part of this paper, examples of new types of Open Collaboration in our group are introduced, and one of them is explained in more detail in a separate section.

\subsection{Examples of new types of Open Collaboration}

\subsubsection{Example \#1}

This is a project to develop a new optical MEMS as a future key device for a customer's main business. The performance which the company wanted to demonstrate was clear but challenging. We have a lot of experience with the technology related to this device, and thus the customer selected us as its R\&D partner rather than studying it internally. We organized the supply chain of this project with other organizations to provide the best proposal to the 
customer. The design, a part of the fabrication and the final evaluation of the device were carried out by us; most of the fabrication and measurement were carried out by MEMS Core (Sendai, Japan), a local MEMS foundry; and the material preparation was carried out by two other companies. The customer just signed a contract with us, and the project was managed by us. The correct choice of required technologies and the organization of the skilled R\&D team across different organizations would have needed a longer time and eventually cost more for the customer. The customer was satisfied with the outcome and the method of R\&D.

\subsubsection{Example \#2}

This is a project to develop a new thin film material for a customer's future device. This material is synthesized by a deposition tool, which was developed by us under "Creation of Innovation Centers for Advanced Interdisciplinary Research Areas Program" funded by MEXT (2007-2016). The customer purchased their own tool from Technofine (Sendai, Japan), a local small tool company where my former student is working, and installed it in Microsystem Integration Center. The R\&D was carried out using this tool by my former student under the supervision of our senior researcher. This $\mathrm{R} \& \mathrm{D}$ was difficult using standard tools commercially available, and the development of a special tool was necessary but would have needed a longer time and cost more for the customer.

\section{MEMS-LSI Integrated Tactile Sensor (Example \#3)}

\subsection{Project scheme}

Under Creation of Innovation Centers for Advanced Interdisciplinary Research Areas Program, Prof. Masayoshi Esashi initiated a project on an integrated tactile sensor for humanfriendly robots with project member companies, and younger researchers including myself have took over the project several years ago. This project demonstrates good practice of Open Collaboration, and is thus explained in this section. Robots working with humans in factories, daycare centers, hospitals and homes are expected in the coming super-aged society with a decline in the labor force population. Tactile sensors covering robot bodies are useful for safe and gentle physical communication as well as skilled manipulation based on machine learning.

Our tactile sensor is MEMS-based and integrated with an LSI, which offers analog frontend, signal processing, bus communication and other smart functions. The development of this sensor requires a lot of resources especially for the design and manufacturing of the LSI. In addition, the system for the readout and control of the sensors must be developed in parallel by an interdisciplinary team. On the other hand, the market for human-friendly robots is waiting to be developed and difficult to predict. Therefore, Open Collaboration is suitable for this project. The project team is organized by us, Toyota Motor (Toyota, Japan), Toyota Central R\&D Labs (Nagakute, Japan), and Toppan Technical Design Center (Tokyo, Japan). This project is open also in another sense. Other companies which can supplement the supply chain of the tactile sensor are welcome. For example, we need a supplier and manufacturer of the tactile sensor. 
Even new users of the tactile sensor are welcome, because the number of the tactile sensors used by Toyota Motor is too small for a device supplier's business.

\subsection{Technical overview}

We assume that a lot of tactile sensors are used in a robot in the future. The number and pitch of the tactile sensors depend on where they are used, e.g. hand, arm, body or leg. A key issue of such applications is how to construct a large-scale network of the tactile sensors with the minimum amount of wiring. Our solution is to use a bus network and "sensor platform LSIs", as shown in Fig. 1.

The sensor platform LSI has functions of multi-axis capacitive and resistive sensor readout, signal processing, bus communication, configuration and so forth. ${ }^{(1)}$ In addition, it asynchronously works in event-driven mode, and thus the first response of the tactile sensors is fast regardless of the number of the tactile sensors connected. ${ }^{(2)}$ When an input signal exceeds the threshold, which is configurable via the bus, the tactile sensor is "excited" and transmits a data packet to the bus similarly to neurons. This event-driven mode also contributes to the reduction in the number of data packets on the bus which is shared by many devices.

We have developed two types of integrated tactile sensors. Figure 2 shows the first type of tactile sensor. ${ }^{(3,4)}$ The LSI wafer and a special low temperature cofired ceramic (LTCC) via wafer ${ }^{(5)}$ are bonded and interconnected by $\mathrm{Au}-\mathrm{Au}$ bonding. ${ }^{(6)}$ The diaphragm is fabricated in the LSI wafer by deep reactive ion etching (DRIE) after bonding. The size of the LTCC
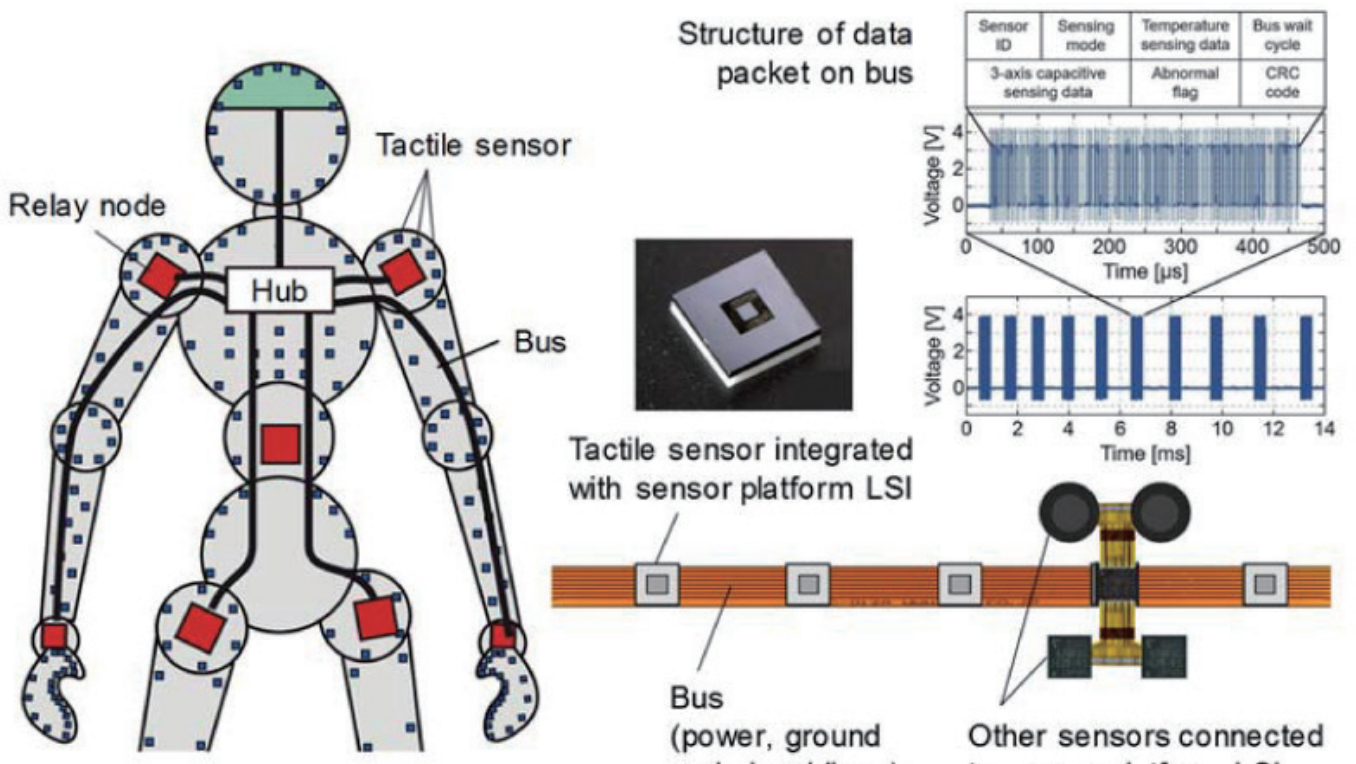

Tactile sensor integrated

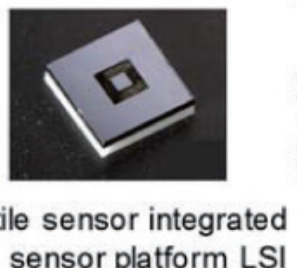
with sensor platform LSI

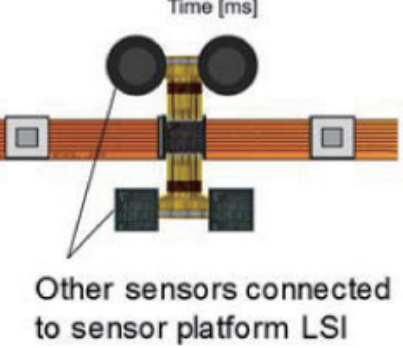

Fig. 1. (Color online) Bus-networked tactile sensor for robots. 

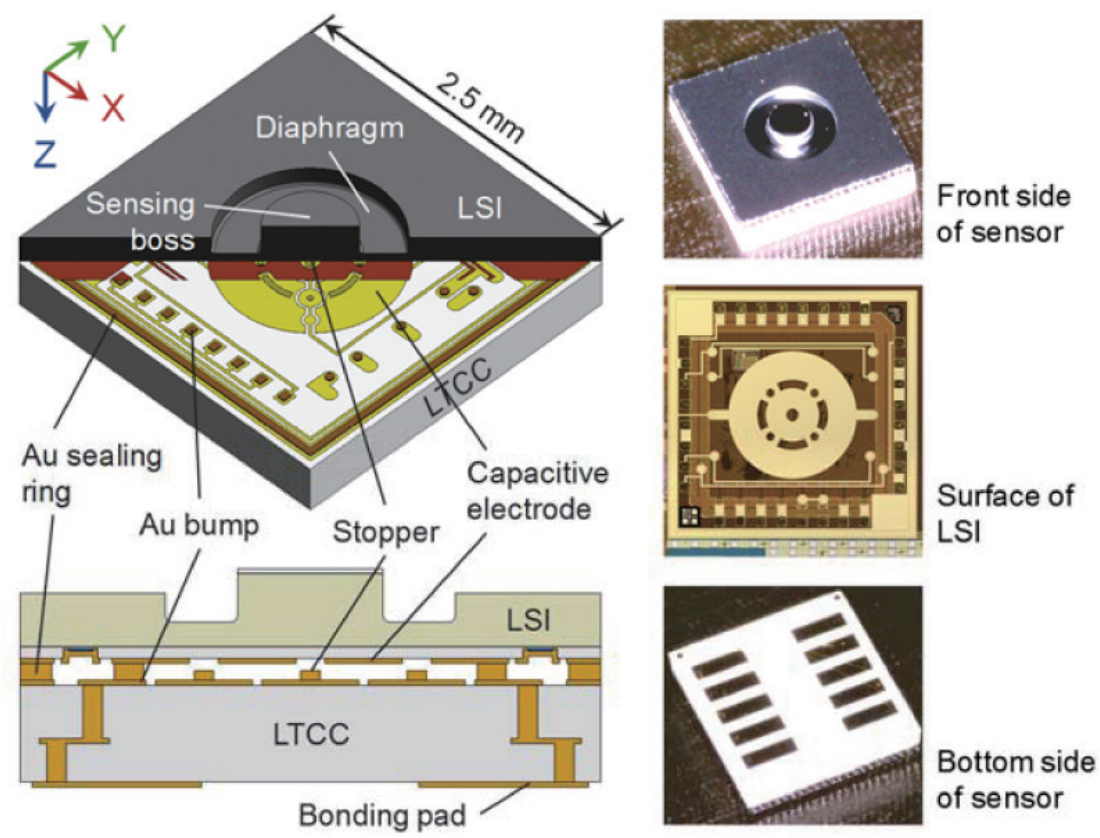

Fig. 2. (Color online) Integrated tactile sensor using sensor platform LSI flip-chip-bonded with LTCC substrate. ${ }^{(4)}$

wafer is limited to $100 \mathrm{~mm}$ in diameter, and thus the LSI wafer of 200 or $300 \mathrm{~mm}$ diameter is trimmed into $100 \mathrm{~mm}$ wafers or even smaller. This is not good for large-volume production but convenient for small-volume production in conjunction with the simple structure and fabrication process of this tactile sensor. The residual part of the LSI wafer after the trimming process will be sold as a discrete application-specific standard product (ASSP), because the LSI can be used as an interface for various sensors. ${ }^{(7)}$

The second type of tactile sensor uses through-silicon vias (TSVs) in the sensor platform LSI, as shown in Fig. $3{ }^{(8)}$ In this project, the LSI was prototyped using a commercial multiproject wafer (MPW) service by TSMC (Hsinchu, Taiwan). The surface of the delivered MPW is laser-erased except for our own die areas to disclose other customers' LSI designs. The TSVs were manufactured in our facility after trimming into $100 \mathrm{~mm}$ wafers, planarizing the lasererased area and backgrinding. The TSV is not filled with $\mathrm{Cu}$ to accommodate the thermal expansion mismatch between $\mathrm{Cu}$ and $\mathrm{Si}$, which may have an impact on reliability owing to a large wafer thickness of $300 \mu \mathrm{m}$. The LSI with the TSVs were integrated with a fully differential 3-axis tactile sensor manufactured by Toyota Central R\&D Labs. ${ }^{(9)}$

The TSV-on-MPW technology ${ }^{(10)}$ is useful for other applications. It is basically open to other customers except direct competitors, although Toyota Group made significant contributions to this R\&D. This is mainly because we want to establish the supply chain of this technology, but it is impossible without Toyota Group's understanding of Open Collaboration. 


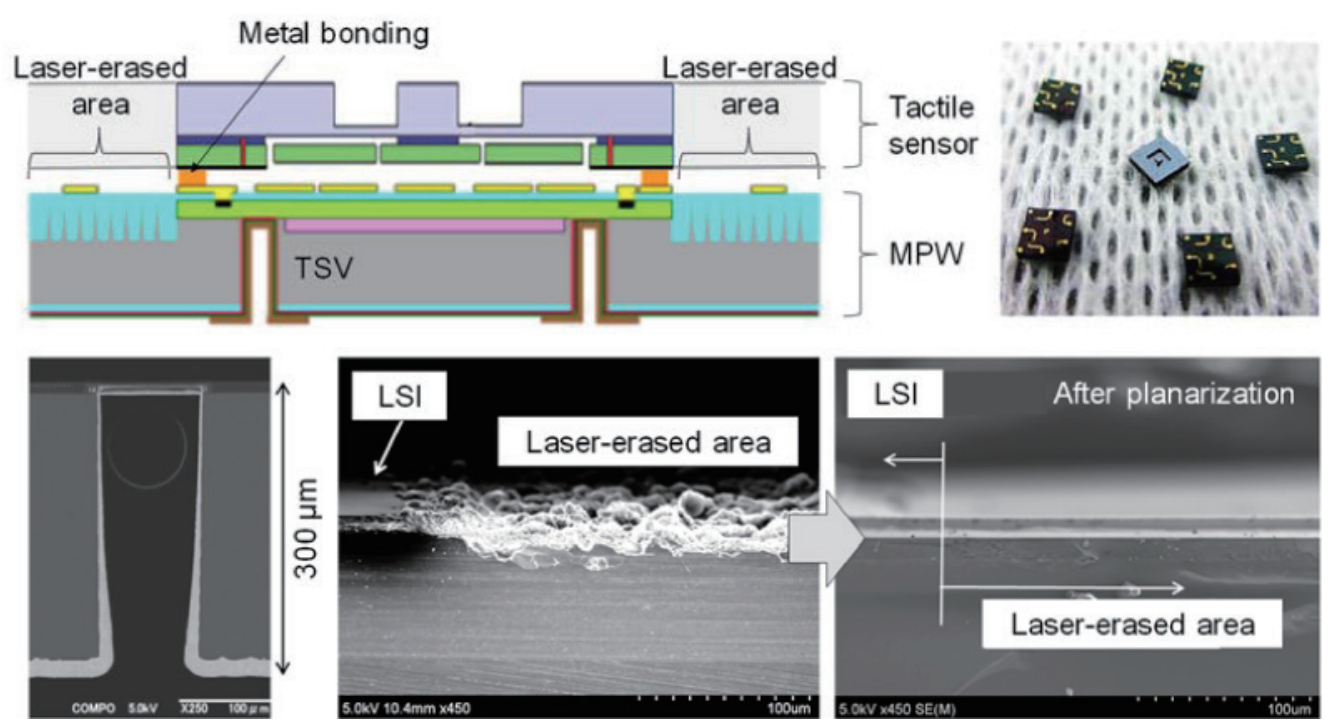

Fig. 3. (Color online) Integrated tactile sensor using sensor platform LSI with TSVs. ${ }^{(9,10)}$

\section{Conclusion}

Open Collaboration is now inevitable as a faster and more cost-effective way to achieve success in the R\&D of MEMS than fully internal R\&D. In the first section, the concept of Open Collaboration was discussed. In my understanding, it is defined as R\&D in a value/ supply chain composed of different organizations including companies, universities and/or research institutes. This is not a new concept but has not worked well in Japanese universities compared with European research institutes. In the second section, the scheme of Open Collaboration in the R\&D division of Microsystem Integration Center and S. Tanaka Laboratory was introduced. We are well experienced in multiple types of Open Collaboration. In the third section, the project of the integrated tactile sensors was explained as a successful example of Open Collaboration. Open Collaboration of MEMS in Tohoku University has been planted and grown by Prof. Masayoshi Esashi, Guest Editor of this special issue, and is now blooming under his successors.

\section{Acknowledgments}

The work described in the third section was supported by "Creation of Innovation Centers for Advanced Interdisciplinary Research Areas Program" under MEXT (2007-2016), and conducted with Toyota Motor, Toyota Central Labs and Toppan Technical Design Center. From 2015, Tohoku University was supported by "Future AI and Robot Technology Development Project" under New Energy and Industrial Technology Development Organization (NEDO) for the commercialization of the tactile sensor and related technologies. 


\section{References}

1 M. Muroyama, T. Nakayama, Y. Hata, Y. Nonomura, T. Bartley, and S. Tanaka: Proc. Smart Systems Integration 2016 (2016) 320.

2 C. Shao, S. Tanaka, T. Nakayama, Y. Hata, and M. Muroyama: Sensors 18 (2018) 231.

3 S. Asano, M. Muroyama, T. Bartley, T. Kojima, T. Nakayama, U. Yamaguchi, H. Yamada, Y. Nonomura, Y. Hata, H. Funabashi, and S. Tanaka: Sens. Actuators, A 240 (2016) 167.

4 S. Asano, M. Muroyama, T. Nakayama, Y. Hata, Y. Nonomura, and S. Tanaka: Sensors 17 (2017) 2451.

5 S. Tanaka, S. Matsuzaki, M. Mohri, A. Okada, H. Fukushi, and M. Esashi: Technical Digest of IEEE MEMS (IEEE, 2011) 376.

6 M. Salman Al Farisi, H. Hirano, J. Frömel, and S. Tanaka: J. Micromech. Microeng. 27 (2017) 015029.

7 C. Shao, S. Tanaka, T. Nakayama, Y. Hata, T. Bartley, Y. Nonomura, and M. Muroyama: Sensors 17 (2017) 1974.

8 Y. Hata, Y. Suzuki, M. Muroyama, T. Nakayama, Y. Nonomura, R. Chand, H. Hirano, Y. Omura, M. Fujiyoshi, and S. Tanaka: Proc. Transducers 2017 (2017) 500.

9 Y. Hata, Y. Nonomura, Y. Omura, T. Nakayama, M. Fujiyoshi, H. Funabashi, T. Akashi, M. Muroyama, and S. Tanaka: Sens. Actuators, A 266 (2017) 24.

10 Y. Suzuki, H. Fukushi, M. Muroyama, Y. Hata, T. Nakayama, R. Chand, H. Hirano, Y. Nonomura, H. Funabashi, and S. Tanaka: Proc. IEEE MEMS (IEEE, 2017) 744. 